\title{
A Low Complexity Linear Moving Average Filtering Technique for PAR Reduction in OFDM Systems
}

\author{
Hassan Ali1 ${ }^{1}$, Raziq Yaqub ${ }^{2}$ \\ ${ }^{1}$ Department of Electrical Engineering and Computing, The University of Newcastle, Callaghan, Australia \\ ${ }^{2}$ Department of Electrical Engineering and Computer Science, Alabama A\&M University, Normal, AL, USA \\ Email: hassan.ali@newcastle.edu.au,raziq.yaqub@aamu.edu
}

How to cite this paper: Ali, H. and Yaqub, R. (2018) A Low Complexity Linear Moving Average Filtering Technique for PAR Reduction in OFDM System. Int. J. Communications, Network and System Sciences, 11, 199-215.

https://doi.org/10.4236/ijcns.2018.1110012

Received: September 18, 2018

Accepted: October 9, 2018

Published: October 12, 2018

Copyright (๑) 2018 by authors and Scientific Research Publishing Inc. This work is licensed under the Creative Commons Attribution International License (CC BY 4.0).

http://creativecommons.org/licenses/by/4.0/

(c) (i) Open Access

\begin{abstract}
In this paper, a linear moving average recursive filtering technique is proposed to reduce the peak-to-average power ratio (PAR) of orthogonal frequency division multiplexing (OFDM) signals. The proposed low complexity technique is analyzed in an oversampled OFDM system and a simple distribution approximation of the oversampled and linearly filtered OFDM signals is also proposed. Corresponding time domain linear equalizers are developed to recover originally transmitted data symbols. Through extensive computer simulations, effects of the new filtering technique on the oversampled OFDM peak-to-average power ratio (PAR), power spectral density (PSD) and corresponding linear equalizers on the frequency selective Rayleigh fading channel transmission symbol-error-rate (SER) performance are investigated. The newly proposed recursive filtering scheme results in attractive PAR reduction, requires no extra fast Fourier transform/inverse fast Fourier transform (FFT/IFFT) operations, refrains from transmitting any side information, and reduces out-of-band radiation. Also, corresponding linear receivers are shown to perform very close to their frequency domain counterparts.
\end{abstract}

\section{Keywords}

Moving Average Filtering, Recursive Filtering, Orthogonal Frequency Division Multiplexing, Peak-to-Average-Power Ratio, Inverse Fast Fourier Transform

\section{Introduction}

Due to high spectral efficiency, immunity to impulse noise, robustness in multipath fading environments and ease of implementation, OFDM has emerged as 
an attractive multicarrier modulation technique for high speed mobile and wireless communication systems. This transmission technique has been adopted in digital video broadcasting [1], digital audio broadcasting [2], asymmetric digital subscriber line (ADSL) [3], wireless local area networks (WLANs) [4] [5] and 4G LTE networks [6]. Also, OFDM combined with mm-wave technology and multiple-input multiple output (MIMO) techniques has been proposed as one of the strong candidates for the physical layer of 5G cellular networks [7] [8] [9] [10].

One major problem associated with OFDM transmitted signal is the high PAR [11] [12], caused by the coherent summation of the OFDM subcarriers. In the extreme case, when all the $N$ subcarriers are added with the same phase, the OFDM signal can have a PAR of about $N$. Such a high PAR causes saturation in power amplifiers (PAs), which leads to significant spectral spreading and in-band distortion [13] [14]. In addition, a high PAR leads to additional bits requirement in the digital-to-analogue and analogue to-digital converters (DACs and ADCs) [15], which can have a significant influence on the power consumption, sensitivity and SER performance at the receiver side.

To alleviate the PAR problem in OFDM systems, several approaches have been devised. For example, selective mapping (SM) [16] [17] [18], partial transmit sequence (PTS) [19] and their hybrid [20] techniques are effective in reducing the PAR with no in-band distortion and simple symbol recovery at the receiver but involve heavy computational load due to many IFFT operations and a complex optimization process. Also, the side information about the phase factors should be transmitted to the receiver for the inverse operation, which not only results in significant data rate loss with increase in the number of carriers but can degrade receiver performance if received erroneously. In [21] [22] a class of distortion less methods for PAR reduction using some virtual subcarriers have been proposed. These schemes offer significant amount of PAR reduction at the cost of a computationally intensive optimization process. Furthermore, since these methods rely on the presence of un-used subcarriers for PAR reduction, they are suitable for discrete multi-tone (DMT) systems. One class of distortionless PAR reduction techniques involves altering modulation constellations to reduce large signal peaks at the cost of increased signal power and many complex IFFT/FFT operations to achieve improved performance [23] [24]. The increase in transmit signal power has a positive effect on the SER at the receiver. However, when the transmit power is fixed these techniques cause SER performance degradation. Another class of distortionless PAR reduction methods constitute systematic coding techniques [25] [26] [27] that can deterministically bound the PAR with little computational cost at the transmitter but the PAR versus data rate trade-off is not attractive.

Deliberate amplitude clipping of the OFDM signal [28] could be the most simple and efficient way of PAR reduction but being a non-linear technique may cause significant in-band distortion, which degrades SER performance and spectral spreading, which reduces spectral efficiency [14]. Filtering after clipping can reduce the spectral splatter [29]; however, the nonlinear in-band distortion can- 
not be corrected through linear receivers. Therefore, iterative clipping noise cancelation techniques are used at the receiver side, which not only rely on coding but also involve several complex FFT/IFFT operations [29] [30] [31].

In this paper, we propose a low complexity moving average PAR reduction filtering technique. We call the technique post-IFFT filtering (PoF) because filtering is applied after IFFT at the transmitter. In the analysis of the proposed technique oversampling of original OFDM signals is considered and a simple mathematical expression to evaluate the complementary cumulative density function (CCDF) of oversampled and linearly filtered OFDM signals is presented.

The proposed technique is simple to implement, requires no extra IFFTs, and refrains from transmitting any side information. The low pass feature of the technique is effective in reducing the out-of-band radiation and thus lends itself to minimize the possible increase in PAR, SER and bandwidth due to pulse shaping filters [11]. Linear zero-forcing (ZF) and minimum-mean square-error (MMSE) equalizers are also developed to compensate for filtering and frequency selective propagation distortions and recover transmitted data symbols. The proposed filtering and equalization schemes can be implemented with any modulation, coding and number of carriers.

This paper is organized as follows: Section 2 characterizes the OFDM transmission and proposes an approximate expression for the distribution of oversampled and linearly filtered OFDM signals. Section 3 covers the preliminaries of the moving average filter. To lay out the groundwork for developing the new technique, the matrix data model of the oversampled OFDM signal is described in Section 4. PoF implementation and corresponding time domain linear symbol recovery solutions are described in Section 5. Several practical issues and pertinent complexity and receiver performance trade-offs are considered in Section 6. In Section 7, illustrating simulations are carried out while conclusions are drawn in Section 8.

\section{OFDM Transmission and PAR Distribution}

In OFDM transmission, a high speed input bit stream, after complex modulation mapping, is converted into complex data symbols of length $N$, which is transformed into an OFDM signal via $N$-point IFFT. The resulting continuous time complex baseband OFDM signal $x_{a}(t)$ can be written as

$$
x_{a}(t)=x_{a}(t)^{I}+j x_{a}(t)^{Q}=\frac{1}{\sqrt{N}} \sum_{k=-N / 2}^{N / 2-1} s(\langle k+N\rangle) \mathrm{e}^{\frac{j 2 \pi k t}{T_{s}}}
$$

where $0 \leq t \leq T_{s}, \quad x_{a}(t)^{I}$ and $x_{a}(t)^{Q}$ denote the real and imaginary parts of $x_{a}(t), j^{2}=-1,\langle k+N\rangle$ denotes $(k+N)$ modulo $N$, $\boldsymbol{s}_{N}=[s(0), s(1), \cdots, s(N-1)]^{\mathrm{T}}$ represents the size $N$ complex input data symbol, $s(k)$ represents the complex modulated symbol of the $k$ th subcarrier, $N$ is the number of subcarriers, and $T_{s}$ denotes the symbol period of the OFDM signal. A cyclic prefix (CP) (i.e., guard interval) is added to the resulting signal in 
order to avoid the inter-block-interference (IBI) in time dispersive channels. The $\mathrm{CP}$ does not affect the PAR characteristics to be analyzed in this paper. Therefore, in order not to complicate the notation, the $\mathrm{CP}$ has been omitted here.

The PAR of $x_{a}(t)$ can be defined as

$$
\varsigma_{a} \triangleq \frac{\max _{0 \leq t \leq T_{s}}\left|x_{a}(t)\right|^{2}}{P_{a v}}
$$

where $P_{a v}$ is the average power defined as $P_{a v} \triangleq E\left\{\left|x_{a}(t)\right|^{2}\right\}=\frac{1}{T_{s}} \int_{0}^{T_{s}}\left|x_{a}(t)\right|^{2} \mathrm{~d} t$ and $P_{a v}=\left\{|s(k)|^{2}\right\}$ based on Parseval's theorem.

The Nyquist rate samples of the OFDM waveforms (1) (i.e., $\left.\left.x_{a}(t)\right|_{t=n T_{s} / N}\right)$ can be represented as:

$$
\breve{x}(n)=\breve{x}(n)^{I}+\breve{j}(n)^{Q}=\frac{1}{\sqrt{N}} \sum_{k=-N / 2}^{N / 2-1} s(\langle k+N\rangle) \mathrm{e}^{\frac{j 2 \pi n k}{N}}
$$

where $0 \leq n \leq N-1$, and the real and imaginary parts of $\breve{x}(n)$ are denoted by $\breve{x}(n)^{I}$ and $\breve{x}(t)^{Q}$, respectively. The PAR of $\breve{x}(n)$ can thus be defined as

$$
\varsigma \triangleq \frac{\max _{0 \leq n \leq N-1}|\breve{x}(n)|^{2}}{P_{a v}}
$$

Based on (3), a simple approximate expression for the distribution of the PAR has been derived in [11]. The input information symbols are assumed to be statistically independent and identically distributed (i.i.d.) with zero mean and variance $\sigma_{s}^{2}=E\left\{|s(k)|^{2}\right\}$. So, when $N$ is large (i.e., $N \geq 64$ ), the samples $\breve{x}(n)$ in (3) are mutually uncorrelated and consequently the $\breve{x}(n)^{I}$ and $\breve{x}(t)^{Q}$, are i.i.d. Gaussian random variables with zero mean and common variance $E\left\{|s(k)|^{2}\right\} / 2=\sigma_{s}^{2} / 2$, according to the central limit theorem. This allows samples $\breve{x}(n)$ in (3) to be i.i.d. Gaussian random variables. The corresponding envelope of the OFDM signal $r_{n}=|\breve{x}(n)|$ is therefore Rayleigh distributed, while the power $p_{n}=r_{n}^{2}$ distribution becomes a central chi square distribution. The PAR CCDF can then be derived and is given by

$$
\operatorname{Pr}\left(\varsigma>\varsigma_{0}\right) \approx 1-\left(1-\mathrm{e}^{-\varsigma_{0}}\right)^{N}
$$

To approximate more accurately the PAR in (2), an oversampled version of (1) can be used [32]. This can be written as

$$
x(n)=\frac{1}{\sqrt{N}} \sum_{k=-N / 2}^{N / 2-1} s(\langle k+N\rangle) \mathrm{e}^{\frac{j 2 \pi n k}{M}}
$$

where $0 \leq n \leq M-1, M=J N$ and $J>1$ is the oversampling factor. Usually, $J \geq 4$ is used to capture the peaks of $x_{a}(t)$.

The assumption in (5), that the samples are mutually independent and uncorrelated, is not strictly correct when oversampling is applied. Hence, an approximation was proposed in [11] by assuming that the distribution of $N$ subcarriers and oversampling can be approximated by the distribution of $\alpha N$ subcarriers 
without oversampling, with $\alpha$ determined by computer simulations to be 2.8 for $N>64$. The CCDF of the PAR is then given by

$$
\operatorname{Pr}\left(\varsigma>\varsigma_{0}\right) \approx 1-\left(1-\mathrm{e}^{-\varsigma_{0}}\right)^{\alpha N}
$$

In order to reduce the PAR of OFDM signals, we introduce a linear moving average filter before adding the CP. If the complex Gaussian baseband signal (6) is passed through the proposed linear filter, the output is also Gaussian. So, the envelope of the complex OFDM signal after simple filtering has the Rayleigh distribution. However, correlation of the output signal samples will increase due to filter memory; hence the approximation (7) is not valid. This difficulty can be neatly resolved by a similar assumption as for the oversampling case above. We thus propose an empirical approximation by assuming that the distribution of $N$ carriers with oversampling and filtering can be given by $\alpha \beta N$ subcarriers, without oversampling and filtering. Consequently, the CCDF of PAR reduced OFDM signal can be given by

$$
\operatorname{Pr}\left(\varsigma>\varsigma_{0}\right) \approx 1-\left(1-\mathrm{e}^{-\varsigma_{0}}\right)^{\alpha \beta N}
$$

where $\beta$ is greater than one and can be determined by exhaustive computer simulations. We remark here that straightforward application of (8) is complicated to work with as the parameter $\beta$ depends not only on the filter length $L_{f}$ but also on the OFDM signal size $M$.

\section{Moving Average Filter}

A moving average filter [33] is one of the simplest type of linear-time-invariant (LTI) processor, which is commonly used in digital-signal-processing (DSP) to remove interference, or additive white Gaussian noise (AWGN) from a relatively slowly varying signal. The filter can be described with the finite impulse response (FIR)

$$
h_{f}(n)=\left\{\begin{array}{lc}
1 / L_{f} & 0 \leq n \leq L_{f}-1 \\
0 & \text { otherwise }
\end{array}\right.
$$

where $L_{f}$ is the filter length. Using (6) as input to the filter, the output of the filter can be expressed as

$$
\begin{aligned}
y(n) & =h_{f}(n) * x(n)=\sum_{l=0}^{L_{f}-1} h_{f}(l) x(n-l) \\
& =\frac{1}{L_{f}}[x(n)+\underbrace{x(n-1)+\cdots+x\left(n-L_{f}+1\right)}_{\text {ICI }}]
\end{aligned}
$$

Notice the smoothing window operation performed by the filter. Each smoothed value is computed as the average of a number of preceding data values and the degree of smoothing increases with $L_{f}$. The filtering output (10), contains interfering terms from other subcarriers, the so-called inter-carrier-interference (ICI) effect.

This loss of orthogonality due to ICI is an undesirable effect and is well known to result in SER degradation at the receiver side. 
The filter can be described in the frequency domain by the Fourier transform of the rectangular pulse

$$
H_{f}(\omega)=\frac{1}{L_{f}} \sum_{l=0}^{L_{f}-1} \mathrm{e}^{-j \omega l}=D_{L_{f}}(\omega) \mathrm{e}^{-j \omega\left(L_{f}-1\right) / 2}
$$

where $D_{L_{f}}(\omega)$ is the Drichlet function [33] defined by

$$
D_{L_{f}}(\omega) \triangleq \frac{\sin \left(\omega L_{f} / 2\right)}{L_{f} \sin (\omega / 2)}
$$

Figure 1 illustrates the magnitude and phase plots of the frequency response of the 32-point moving average filter. The filter shows a linear phase and low pass characteristic. The main lobe is not rectangular and there are substantial side lobes. Hence, this FIR filter is a poor approximation of the ideal filter. The width of the main lobe is inversely proportional to $L_{f}$. If $L_{f}$ is large, the shape of $\left|H_{f}(\omega)\right|$ tends to be a sinc function envelope, with significantly reduced sidelobes. It is thus clear that good smoothing performance results in bad low pass filtering performance.

\section{Oversampled OFDM Data Model}

Let the samples associated with the $i$ th data vector of size $M$ be denoted as $x_{M, i}(n)$. With this notation, we can now express the $t$ th oversampled OFDM signal (6) as

$$
\boldsymbol{x}_{M}(i)=\left[x_{M, i}(0), \cdots, x_{M, i}(M-1)\right]^{T}
$$

The vector $\boldsymbol{x}_{M}(i)$ can be obtained by using a $M$-point IFFT on the extended data vector $\boldsymbol{u}_{M}(i)=\left[s_{0}(i), \cdots, s_{N / 2-1}(i), 0, \cdots, 0, s_{N / 2}(i), \cdots, s_{N-1}(i)\right]^{T}$ obtained by inserting $M-N$ zeros in the middle of the information symbol vector $\boldsymbol{s}_{N}(i)=\left[s_{N, i}(0), \cdots, s_{N, i}(N-1)\right]^{T} \quad$ [34]. To describe how the $M-N$ zeros are inserted, we define the $M \times N$ matrix

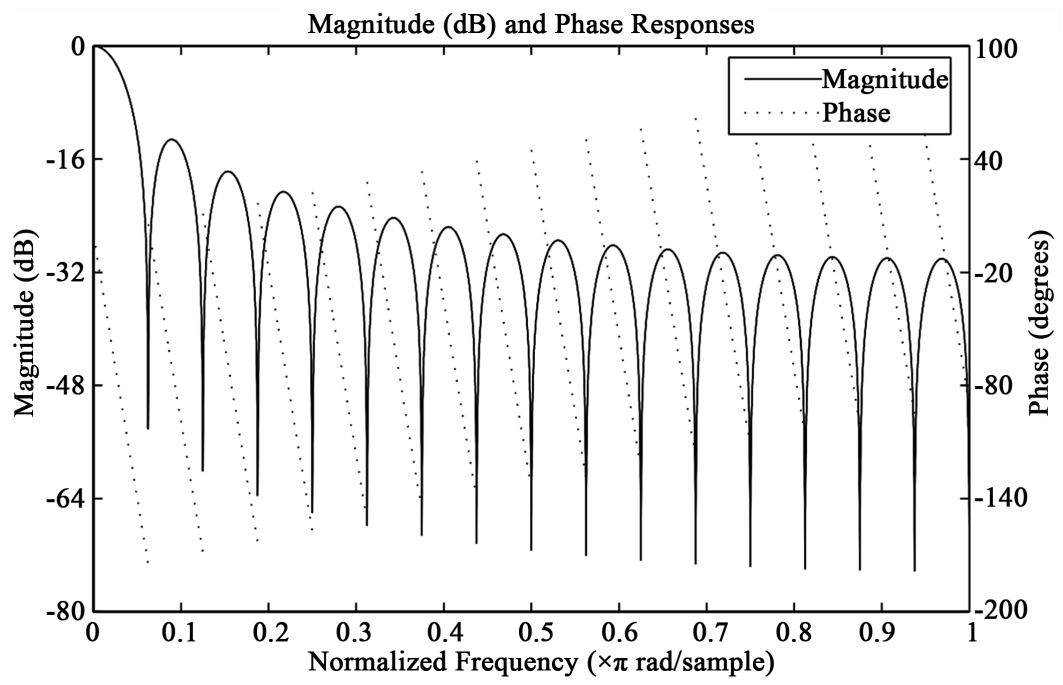

Figure 1. Magnitude and phase response plots of the 32-point moving average filter. 


$$
\boldsymbol{P} \triangleq\left[\begin{array}{cc}
\boldsymbol{I}_{N / 2 \times N / 2} & \boldsymbol{O}_{N / 2 \times N / 2} \\
\boldsymbol{O}_{(M-N) \times N / 2} & \boldsymbol{O}_{(M-N) \times N / 2} \\
\boldsymbol{O}_{N / 2 \times N / 2} & I_{N / 2 \times N / 2}
\end{array}\right]
$$

Pre-multiplying the data vector $\boldsymbol{s}_{N}(i)$ by the precoding matrix $\boldsymbol{P}$ yields the extended data vector $\boldsymbol{u}_{M}(i)=\boldsymbol{P}_{\boldsymbol{s}_{N}}(i)$. The relationship between $\boldsymbol{x}_{M}(i)$ and $\boldsymbol{s}_{N}(i)$, and thus the associated data matrix model for the signal $x_{M, i}(n)$ can be written as:

$$
\boldsymbol{x}_{M}(i)=\gamma \boldsymbol{F}_{M}^{\mathcal{H}} \boldsymbol{u}_{M}(i)=\gamma \boldsymbol{F}_{M}^{\mathcal{H}} \boldsymbol{P} \boldsymbol{s}_{N}(i)
$$

where $\boldsymbol{F}_{M}$ is the $M \times M$ FFT matrix with $(m, n)$ entry $(1 / \sqrt{M} \exp (-j 2 \pi m n / M))$, and the power loss factor $\gamma=\sqrt{N / M}$ is used here to retain the same power before and after the oversampling.

\section{The Post IFFT Filtering (PoF) Technique and Symbol Recovery}

If the oversampled OFDM signals are received by an FIR moving average filter $\boldsymbol{h}_{f}=\left[h_{f}(0), h_{f}(1), \cdots, h_{f}\left(L_{f}-1\right)\right]^{T}$, with $1<n \leq M$, the expression for the $i$ th filter output symbol block can be written as

$$
\breve{\boldsymbol{y}}_{M}(i)=\boldsymbol{H}_{M}\left(\boldsymbol{h}_{f}\right) \boldsymbol{x}_{M}(i)+\tilde{\boldsymbol{H}}_{M}\left(\boldsymbol{h}_{f}\right) \boldsymbol{x}_{M}(i-1)
$$

where $\boldsymbol{H}_{M}\left(\boldsymbol{h}_{f}\right)$ is the $M \times M$ lower triangular Toeplitz filtering matrix with first column $\left[h_{f}(0), \cdots, h_{f}\left(L_{f}-1\right), 0, \cdots, 0\right]^{T}$ and $\tilde{\boldsymbol{H}}_{M}\left(\boldsymbol{h}_{f}\right)$ is the $M \times M$ upper triangular Toeplitz matrix with first row $\left[0, \cdots, 0, h_{f}\left(L_{f}-1\right), \cdots, h_{f}(1)\right]$.

Due to the filter memory, IBI arises between successive blocks and renders $\breve{\boldsymbol{y}}_{M}(i)$ in (16) dependent on both $\boldsymbol{x}_{M}(i)$ and $\boldsymbol{x}_{M}(i-1)$. To avoid IBI and thus to process data blocks independently at the transmitter (and also at the receiver), we assume that all input data prior to $x_{M, i}(0)$ is zero, and make no assumptions about the input data after $x_{M, i}(M-1)$. That way, we can write the expression for the IBI free $i$ th output symbol block as

$$
\boldsymbol{y}_{M}(i)=\left[y_{M, i}(0), \cdots, y_{M, i}(M-1)\right]^{T}=\boldsymbol{H}_{M}\left(\boldsymbol{h}_{f}\right) \boldsymbol{x}_{M}(i)
$$

Notice that

$$
\begin{aligned}
& y_{M, i}(0)=x_{M, i}(0) / L_{f}, \\
& y_{M, i}(1)=\left(x_{M, i}(0)+x_{M, i}(1)\right) / L_{f}, \\
& \quad \vdots \\
& y_{M, i}(M-1)=\left(x_{M, i}\left(M-L_{f}\right)+\cdots+x_{M, i}(M-1)\right) / L_{f}
\end{aligned}
$$

Therefore, a tremendous advantage of this filtering implementation is its efficient recursion

$$
y_{M, i}(n)=y_{M, i}(n-1)+\frac{1}{L_{f}}\left[x_{M, i}(n)-x_{M, i}\left(n-L_{f}\right)\right]
$$

As compared with the matrix vector product (17), the recursion (18) is much 
faster, requiring fewer additions/subtractions regardless of the filter length $L_{f}$. It is not necessary at the transmitter to wait for all the samples of an OFDM symbol before the first filtered outputs are produced. Of course, this is ideal for delay limited systems.

The OFDM signals based on the system model (17) are cyclically extended, digital-to-analogue (D/A) conversion and transmit filtering are performed, the signal is modulated, power amplified and then transmitted through the channel. At the receiver, following the frequency down-conversion, receive filtering and analogue-to-digital (A/D) conversion, the CP of the subsequent OFDM symbols are discarded. We use a discrete time length $L_{c}$, FIR filter with impulse response $\boldsymbol{h}_{c}=\left[h_{c}(0), h_{c}(1), \cdots, h_{c}\left(L_{c}-1\right)\right]^{T}$ to represent the overall combined effect of the spectral shaping pulse, the continuous time channel, the receive filter and the sampling. At the output of the demodulator, the time domain received baseband data vector can be written as:

$$
\boldsymbol{r}_{M}(i)=\boldsymbol{C}_{M}\left(\boldsymbol{h}_{c}\right) \boldsymbol{y}_{M}(i)+\boldsymbol{n}_{M}(i)=\gamma \boldsymbol{C}_{M}\left(\boldsymbol{h}_{c}\right) \boldsymbol{H}_{M}\left(\boldsymbol{h}_{f}\right) \boldsymbol{F}_{M}^{\mathcal{H}} \boldsymbol{P} \boldsymbol{s}_{N}(i)+\boldsymbol{n}(i)
$$

where the FIR channel vector $\boldsymbol{h}_{c}$ is denoted by the $M \times M$ circular channel matrix $\boldsymbol{C}_{M}\left(\boldsymbol{h}_{c}\right)$ with first row $\left[h_{c}(0), 0, \cdots, 0, h_{c}\left(L_{c}-1\right), \cdots, h_{c}(1)\right]$ and $\boldsymbol{n}_{M}(i)=\left[n_{0}(i), n_{1}(i), \cdots, n_{M-1}(i)\right]^{T}$ is the complex AWGN vector.

Since the filtering removes the subcarrier orthogonality at the transmitter, therefore, the data model based on FFT of (19) does not allow us to use standard frequency domain equalizers. We therefore suffice on the time domain data model (19) for symbol recovery. According to the ZF criterion, the equalizer is chosen to assure perfect symbol recovery in the absence of noise. In Equation (19), the matrices $\boldsymbol{P}, \boldsymbol{H}_{M}\left(\boldsymbol{h}_{f}\right)$ and $\boldsymbol{F}_{M}^{\mathcal{H}}$ are full rank by design. Therefore, by assuming that the matrix $\boldsymbol{H}_{M}\left(\boldsymbol{h}_{c}\right)$ is full rank, the ZF solution is unique and is given by $\hat{\boldsymbol{s}}_{N}(i)=\boldsymbol{G}_{\mathrm{zf}}^{\mathrm{PoF}} \boldsymbol{r}_{M}(i)$, where $\boldsymbol{G}_{\mathrm{zf}}^{\mathrm{PoF}}$ is the ZF equalizing matrix, which can be found in two steps. First, we obtain the estimate of $\boldsymbol{y}_{M}(i)=\gamma \boldsymbol{H}_{M}\left(\boldsymbol{h}_{f}\right) \boldsymbol{F}_{M}^{\mathcal{H}} \boldsymbol{P} \boldsymbol{s}_{N}(i)$ as $\hat{\boldsymbol{y}}_{M}(i)=\left(\boldsymbol{C}_{M}\left(\boldsymbol{h}_{f}\right)\right)^{-1} \boldsymbol{r}_{M}(i)$; and then we find $\hat{\boldsymbol{s}}_{N}(i)=\left(\gamma \boldsymbol{H}_{M}\left(\boldsymbol{h}_{f}\right) \boldsymbol{F}_{M}^{\mathcal{H}} \boldsymbol{P}\right)^{\dagger} \hat{\boldsymbol{y}}_{M}(i)$, which leads to

$$
\boldsymbol{G}_{\mathrm{zf}}^{\mathrm{PFF}}=\boldsymbol{W}\left(\boldsymbol{C}_{M}\left(\boldsymbol{h}_{c}\right)\right)^{-1}
$$

where $\boldsymbol{W}=\left(\gamma \boldsymbol{H}_{M}\left(\boldsymbol{h}_{f}\right) \boldsymbol{F}_{M}^{\mathcal{H}} \boldsymbol{P}\right)^{\dagger}$ is an $N \times M$ matrix representing pseudo inverse of the overall combined effect of filtering, IFFT modulation and oversampling operations.

At low signal-to-noise ratio (SNR), a vector MMSE equalizer can lead to improved receiver performance. According to MMSE criterion, the equalizer is chosen to minimize the mean square error (MSE) $E\left\{\|\boldsymbol{e}(i)\|^{2}\right\}=E\left\{\left\|\hat{s}_{N}(i)-s_{N}(i)\right\|^{2}\right\}$. The MSE can be written as a function of the equalizing matrix $\boldsymbol{G}$ as:

$$
\begin{aligned}
J(\boldsymbol{G}):= & E\left\{\operatorname{tr}\left[\left(\gamma \boldsymbol{G} \boldsymbol{C}_{M}\left(\boldsymbol{h}_{c}\right) \boldsymbol{H}_{M}\left(\boldsymbol{h}_{f}\right) \boldsymbol{F}_{M}^{\mathcal{H}} \boldsymbol{P}-\boldsymbol{I}_{N}\right) \boldsymbol{s}_{N}(i)+\boldsymbol{G} \boldsymbol{n}_{M}(i)\right]\right. \\
& \left.\times\left[\left(\gamma \boldsymbol{G} \boldsymbol{C}_{M}\left(\boldsymbol{h}_{c}\right) \boldsymbol{H}_{M}\left(\boldsymbol{h}_{f}\right) \boldsymbol{F}_{M}^{\mathcal{H}} \boldsymbol{P}-\boldsymbol{I}_{N}\right) \boldsymbol{s}_{N}(i)+\boldsymbol{G} \boldsymbol{n}_{M}(i)\right]^{\mathcal{H}}\right\}
\end{aligned}
$$


By setting gradient $\nabla_{G} J(\boldsymbol{G})=\mathbf{0}$ and solving for $\boldsymbol{G}$, MMSE equalizing matrix $\boldsymbol{G}=\boldsymbol{G}_{\mathrm{mmse}}^{\mathrm{PoF}}$ (yielding the MMSE estimate $\left.\hat{\boldsymbol{s}}_{N}(i)=\boldsymbol{G}_{\mathrm{mmse}}^{\mathrm{PoF}} \boldsymbol{r}_{M}(i)\right)$ is given by

$$
\boldsymbol{G}_{\mathrm{mmse}}^{\mathrm{PoF}}=\gamma \sigma_{s}^{2} \boldsymbol{V} \boldsymbol{C}_{M}^{\mathcal{H}}\left(\boldsymbol{h}_{c}\right)\left(\sigma_{n}^{2} \boldsymbol{I}_{M}+\gamma^{2} \sigma_{s}^{2} \boldsymbol{C}_{M}\left(\boldsymbol{h}_{c}\right) \boldsymbol{Q} \boldsymbol{C}_{M}^{\mathcal{H}}\left(\boldsymbol{h}_{c}\right)\right)^{-1}
$$

where $\boldsymbol{V}=\left(\boldsymbol{H}_{M}\left(\boldsymbol{h}_{f}\right) \boldsymbol{F}_{M}^{\mathcal{H}} \boldsymbol{P}\right)^{\mathcal{H}}$ and $\boldsymbol{Q}=\boldsymbol{H}_{M}\left(\boldsymbol{h}_{f}\right) \boldsymbol{H}_{M}^{\mathcal{H}}\left(\boldsymbol{h}_{f}\right)$. Furthermore, in deriving (22) it is assumed that the correlation matrices $\boldsymbol{R}_{s s}:=E\left\{\boldsymbol{s}_{N}(i) \boldsymbol{s}_{N}^{\mathcal{H}}(i)\right\}=\sigma_{s}^{2} \boldsymbol{I}_{N}$ and $\boldsymbol{R}_{n n}:=E\left\{\boldsymbol{n}_{M}(i) \boldsymbol{n}_{M}^{\mathcal{H}}(i)\right\}=\sigma_{n}^{2} \boldsymbol{I}_{M}$.

Since the matrix $\boldsymbol{W}$ in Equation (20), and matrices $\boldsymbol{V}$ and $\boldsymbol{Q}$ in Equation (22), are not channel dependent and remain fixed, therefore, they can be pre-computed and straightforwardly embedded in the receiver.

A block diagram of an OFDM system involving the proposed PoF transform and corresponding time domain linear equalizers is illustrated in Figure 2.

\section{Practical Considerations}

\subsection{Average Transmit Power}

The average power of the PoF OFDM signals is

$$
\begin{aligned}
\frac{E\left\{\left\|\boldsymbol{y}_{M}(i)\right\|^{2}\right\}}{M} & =\frac{\operatorname{tr}\left(E\left\{\boldsymbol{y}_{M}(i) \boldsymbol{y}_{M}^{\mathcal{H}}(i)\right\}\right)}{M} \\
& =\frac{\operatorname{tr}\left(E\left\{\boldsymbol{H}_{M}\left(\boldsymbol{h}_{f}\right) \boldsymbol{x}_{M}(i) \boldsymbol{x}_{M}^{\mathcal{H}}(i) \boldsymbol{H}_{M}^{\mathcal{H}}\left(\boldsymbol{h}_{f}\right)\right\}\right)}{M} \\
& =\sigma_{s}^{2}\left[\frac{\operatorname{tr}\left(E\left\{\boldsymbol{H}_{M}\left(\boldsymbol{h}_{f}\right) \boldsymbol{H}_{M}^{\mathcal{H}}\left(\boldsymbol{h}_{f}\right)\right\}\right)}{M}\right] \\
& =\sigma_{s}^{2}\left[\left(\frac{1+2+\cdots+L_{f}}{L_{f}^{2}}+\frac{M-L_{f}}{L_{f}}\right) / M\right]
\end{aligned}
$$

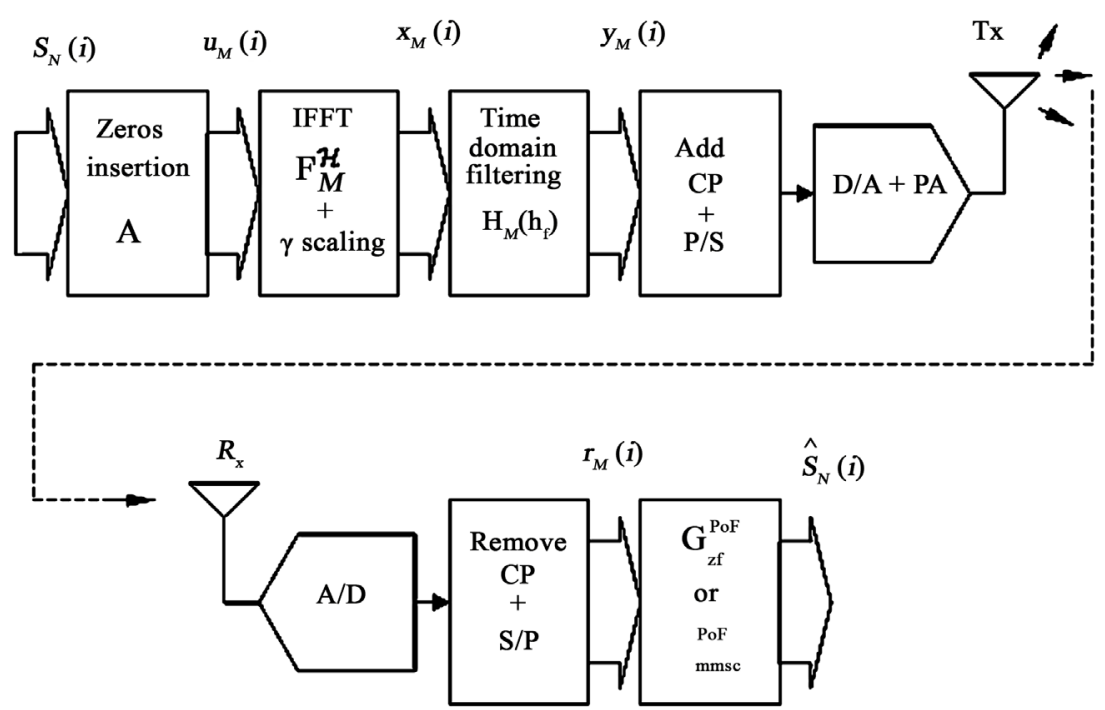

Figure 2. Block diagram of CP-OFDM system with PoF scheme and corresponding linear equalizers. 
The sum of an arithmetic series consisting of $n$ terms $a_{1}, a_{2}, \cdots, a_{n}$ with common difference $d$ is given by $a_{1}+a_{2}+\cdots+a_{n}=n\left(a_{1}+a_{n}\right) / 2$. This implies that $\left(1+2+\cdots+L_{f}\right) / L_{f}^{2}=\left(1+L_{f}\right) / 2 L_{f}$. Therefore (23) can be written as

$$
\frac{E\left\{\left\|\boldsymbol{y}_{M}(i)\right\|^{2}\right\}}{M}=\sigma_{s}^{2} \frac{\left(2 M-L_{f}+1\right)}{2 M L_{f}}
$$

It is thus clear from (23) that the PoF PAR reduction process also involves reduction in the average power of OFDM signals ${ }^{1}$. Apart from PAR, the reduction in average transmit power affects the system performance in two ways, one positive and one negative. Firstly, it will result in a more desirable spectrum. Secondly, it will decrease SNR at the receiver, which means an increase in SER. We will present these simulations in the next section.

\subsection{Oversampling}

The oversampling can be seen to increase the size of IFFT/FFT matrices in the proposed linear transceivers, resulting in increased computational complexity and bandwidth requirements. Though proposed filtering and symbol recovery schemes can straightforwardly work on Nyquist sampled OFDM signals, however, oversampling must be provided in the process to approximate more accurately the PAR and it is for this reason several PAR reduction approaches and corresponding PAR distribution studies based on oversampled OFDM signals have appeared in literature [35] [36] [37]. Indeed, oversampling is already used in practical OFDM systems. The main reason for this is the anti-aliasing filter, which is required to suppress the mirror spectra from the D/A conversion in the analogue OFDM signal $x_{a}(t)$ [38]. Thus, instead of a $N$-point IFFT/FFT an $M$-point IFFT/FFT is actually done in real systems.

\subsection{Complexity}

The simple recursive PoF approach requires only $2 M$ additions per OFDM data vector ( 2 additions per sample). Generally, computing time for one addition is much less than that for one multiplication (which requires 4 real multiplications and 2 real additions). This shows that the PoF technique is a computationally efficient approach.

The matrix-vector product $\boldsymbol{G}^{\mathrm{PoF}} \boldsymbol{r}_{M}(i)$, for obtaining $\hat{\boldsymbol{s}}_{N}(i)$ requires $\mathcal{O}\left(N^{2}\right)$ computations. So the computational complexity per symbol of our linear equalizers in Equations (20) and (22) is $\mathcal{O}(N)$. Of course, this is higher than the standard frequency domain equalizers which have per symbol complexity of order $\mathcal{O}(\log M)$, but computationally much heavier Viterbi like approaches and iterative ${ }^{2}$ techniques involving multiple FFTs to recover symbols are not required.

\footnotetext{
${ }^{1}$ Dependency of the average transmit power on $L_{f}$ and $M$ supports our claim in Section 2 that the parameter $\beta$ depends on $L_{f}$ and $M$.

${ }^{2}$ These techniques are practical only when channel length, symbol size and/or number of carriers are relatively small.
} 


\subsection{Coded Transmissions and Constellation}

In practical OFDM systems, error control codes are usually used to combat channel nulls (or deep fades). Our proposed filtering and symbol recovery approaches are applicable if the coded symbols are transmitted. The methods do not capitalize on any particular type of constellation, hence they are directly applicable to the cases where transmitted information is drawn from any signal constellation.

\subsection{Spectral Nulls}

The time domain ZF equalizer, assures symbol recovery if the circulant matrix $\boldsymbol{C}_{M}\left(\boldsymbol{h}_{c}\right)$ is full rank. The matrix $\boldsymbol{C}_{M}\left(\boldsymbol{h}_{c}\right)$ is full rank if and only if the transfer function $\boldsymbol{h}_{c}$ has no zeros on the FFT grid. Although we may adopt the MMSE equalizer when the channel has nulls on (or close to) the FFT grid, but lack of equalizability will result in an error floor in the resulting SER performance.

\section{Simulations}

In order to verify the performance of the proposed schemes, we consider (unless otherwise specified) a baseband OFDM system with the number of subcarriers $N=80$, the oversampling factor $J=4$, and randomly generated input data are modulated by quaternary phase-shift keying (QPSK). Furthermore, we call proposed time domain equalizers: PoF-ZF and PoF MMSE equalizers for convenience, in the rest of this paper.

Figure 3 shows the CCDFs of PAR for original OFDM, and filtered OFDM signals with varying $L_{f}$, using $10^{5}$ random realizations of corresponding signals. As can be seen, filtering results in a more desirable statistical characteristic.

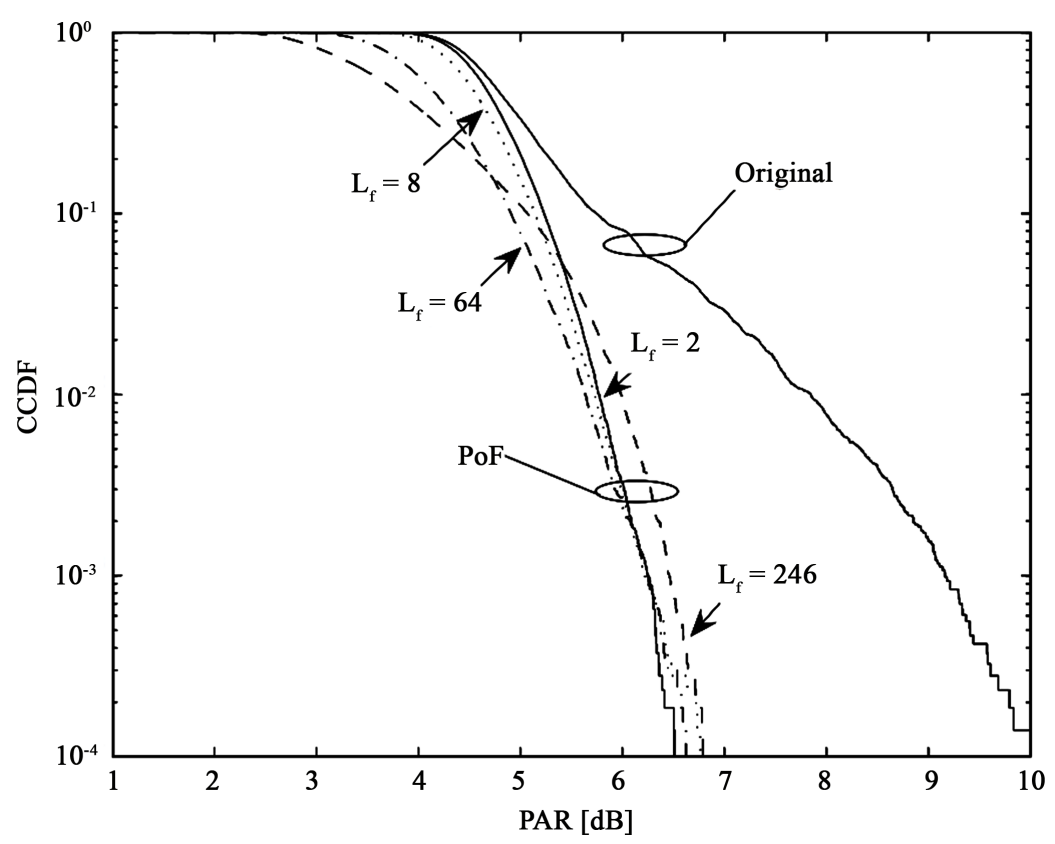

Figure 3. PAR CCDF comparison of original and PoF OFDM signals for varying $L_{f}$ 
At excess probability of $10^{-4}$, the PAR reduction is $3.5 \mathrm{~dB}$ for $L_{f}=2$, whereas, diminishing effect in PAR returns can be observed for $L_{f}>2$. From (23) [or equivalently (24)], $L_{f}=2$ also yields the minimum reduction in the average transmit power. Furthermore, notice that as $L_{f}$ decreases, so does the ICI and corresponding improvement in SNR. This shows that filter with $L_{f}=2$ not only yields better PAR but also will result in better SER performance at the receiver side as compared with higher values of $L_{f}$. We therefore limit suitability of the PoF scheme with $L_{f}=2$. Since $L_{f}=2$ is now fixed, the receiver need not to be notified of the filter length whenever filtering is applied, thereby eliminating the need of side information overhead.

To demonstrate the effectiveness of the low complexity (here called high-speed) filtering option in Equation (18) against the matrix vector product option in Equation (17) (here called direct), Figure 4 shows the direct and high-speed times for $10 \leq M \leq 200$ (thus $40 \leq M \leq 800$ ) and $L_{f}=2$, by executing Matlab script over a $3 \mathrm{GHz}$ Processor. The average execution times are computed over 500 realizations of random signals. It is seen that when the signal length $M$ is small, the difference in performance of both the methods is not noticeable. However, as $M$ becomes large the direct method times increase considerably, while the high-speed convolution computational times increase very slowly. Clearly, the high-speed option is very efficient for practical PAR reduction in OFDM systems with any number of subcarriers.

In Figure 5, we show the PSD (averaged over 500 realizations) of the original and filtered OFDM signals with $L_{f}=2$. Due to filtering of the OFDM signal, the ICI and thus the in-band distortion is evident. It can be observed that no spectral splatter is caused, and out-of-band distortion is reduced. This provides much better operating conditions where non-linear amplifiers are used.

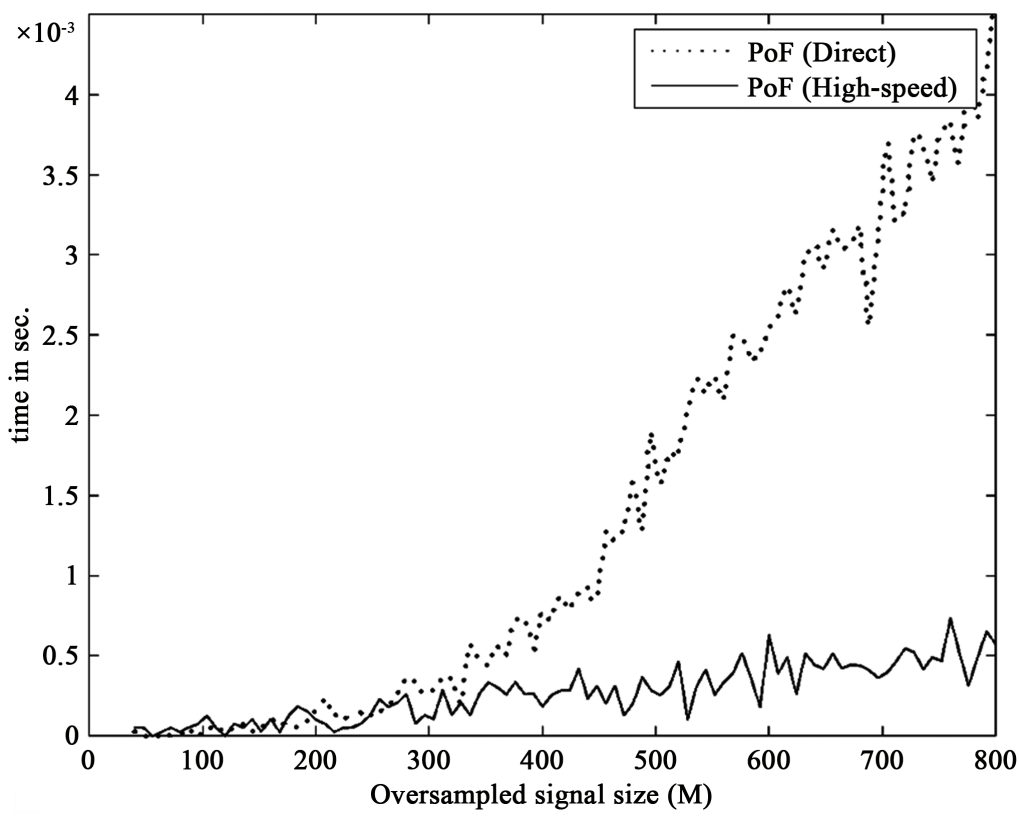

Figure 4. Comparison of direct and high-speed options $\left(L_{f}=2\right)$. 


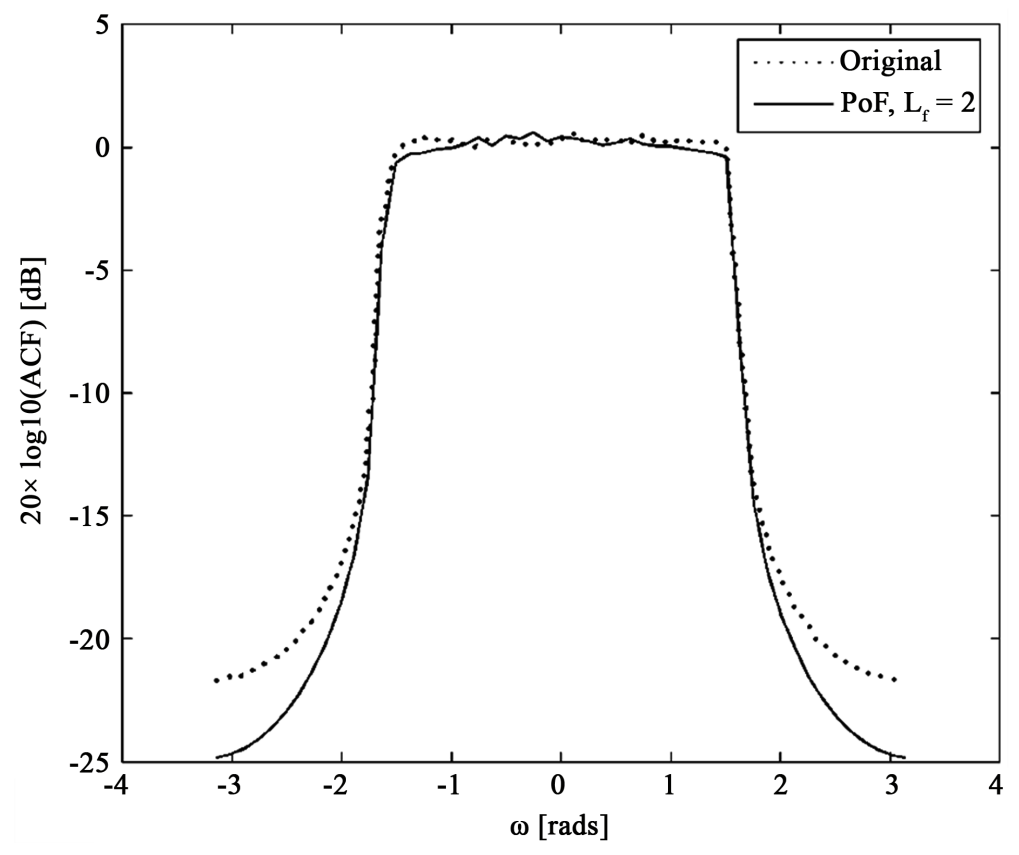

Figure 5. PSD spectra of original and filtered OFDM signals.

The unwanted sideband power is normally reduced through the use of pulse shaping filters [11] [39] [40]. Note that the overall discrete time channel impulse response $\boldsymbol{h}_{c}$ is the convolution of the sampled continuous time: transmit pulse shaping filter, channel and receive filter that is matched with the transmit filter, therefore for a given channel, transmit and receive pulse shaping filters increase the size of $L_{c}$. A large $L_{c}$ not only requires an increase in the CP length, affecting the bandwidth efficiency, but due to ICI also contributes to SER degradation. Furthermore, it may lead to a possible PAR increase [41]. Reduced sidebands due to the proposed scheme allow much better operating conditions for pulse shaping filters. Therefore, pulse shaping filters of smaller length can be used and thus problems related to pulse shaping can be reduced. This is a highly desirable characteristic since many modern communication systems, such as the wireless cellular system, operate in environments where pulse shaping is required for limiting the bandwidth of signals.

Figure 6 shows the SER comparison between the proposed PoF and standard frequency domain ZF and MMSE equalization schemes in a wireless fading environment. The filtered and original OFDM signals were transmitted through a 3-ray quasi-static Rayleigh fading channel of which the first and second fading rays were inactive. The carrier frequency was set at $900 \mathrm{MHz}$ and active ray was assumed to fade at the Doppler frequency of $f_{d}=50 \mathrm{~Hz}$. We assumed that channel was perfectly known to the receiver and the SER performance was evaluated for 1200 randomly generated OFDM symbols. SER performance of the frequency domain ZF and MMSE equalizers (shown as ZF and MMSE in Figure 6), based on oversampled OFDM signal (15), is shown as a bound to benchmark the performance of the proposed PoF equalizers. In both the time and frequency domains, ZF and MMSE equalizers are seen to perform equally (although the 


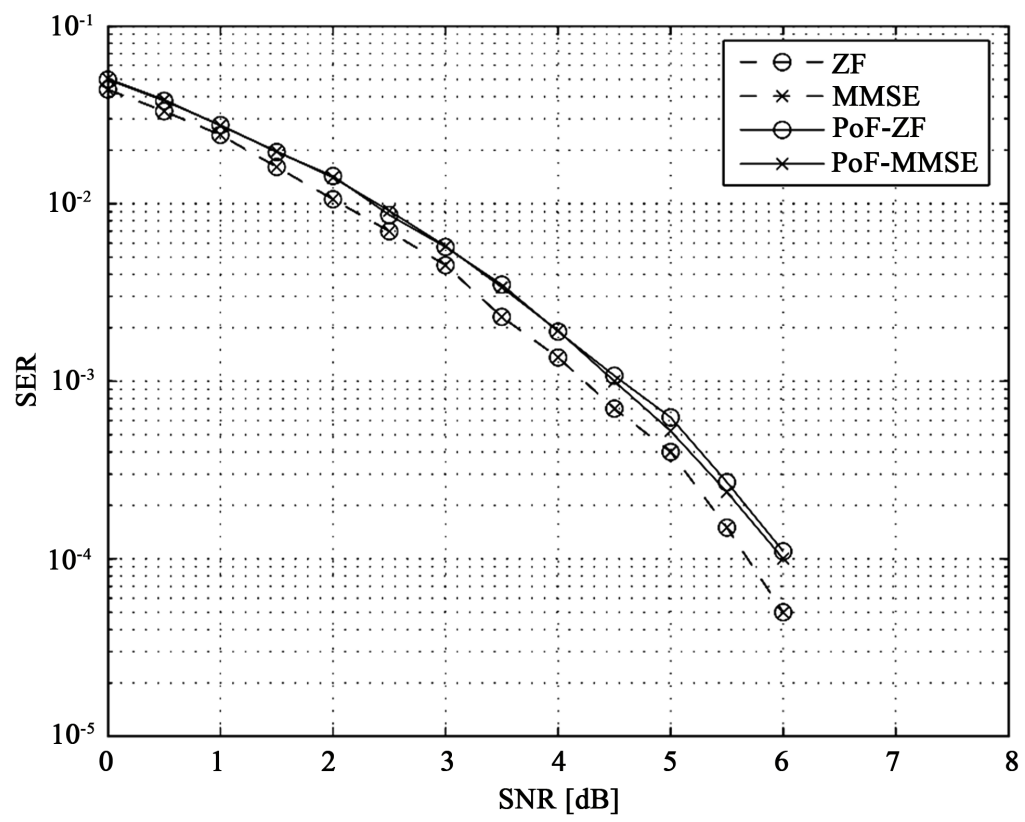

Figure 6. SER comparison between proposed PoF and standard frequency domain equalizers.

later approach offers lower MSE) and all equalizers are able to correct all transmitted symbols above SNR of $6 \mathrm{~dB}$. A performance gap emerges between the PoF and frequency domain equalizers; however, no significant performance loss can be seen due to the filtering operation.

\section{Conclusion}

In this paper, a computationally efficient PAR reduction technique based on linear moving average filtering (that we called PoF) was proposed. We also proposed the distribution function of oversampled and linearly filtered OFDM signals. The proposed filtering technique relies on time domain recursive approach for efficient implementation and requires the filter length $L_{f}=2$. The scheme results in attractive PAR reduction, requires no extra FFT/IFFT operations, and refrains from transmitting any side information. A key feature of the technique is the reduction in out-of-band radiation and problems related to the pulse shaping. The effect of multicarrier modulation, oversampling, filtering and channel dispersion is modelled as a linear transformation. Therefore, to recover the originally transmitted symbols, we proposed corresponding time domain linear ZF and MMSE receivers which were seen to perform very close to their frequency domain counterparts. The new filtering and equalization schemes do not capitalize on a particular coding or constellation technique and can be used for any number of subcarriers.

\section{Conflicts of Interest}

The authors declare no conflicts of interest regarding the publication of this paper. 


\section{References}

[1] European Telecommunications Standards Institute (ETSI) (2000) Digital Video Broadcasting (DVB): Framing Structure, Channel Coding and Modulation for Digital Terrestrial Television. 1st Edition, ETSI, EN 300744.

[2] European Telecommunications Standards Institute (ETSI) (2000) Radio Broadcasting Systems: Digital Audio Broadcasting (DAB) to Mobile, Portable and Fixed Receivers. 1st Edition, ETSI, EN 300401.

[3] European Telecommunications Standards Institute (ETSI) (1997) Asymmetric Digital Subscriber Line (ADSL) Metallic Interface. ANSI, ANSI/TEI/9J-007.

[4] Institute of Electrical and Electronics Engineers (IEEE) (1999) Part II: Wireless LAN Medium Access Control (MAC) and Physical Layer (PHY) Specifications: High-Speed Physical Layer in 5 GHz Band. IEEE Sts. 802.11a-1999.

[5] van Nee, R., Awater, G., Morikura, M., Takanashi, H., Webster, M. and Halford, K. W. (1999) New High Rate Wireless LAN Standards. IEEE Communications Magazine, 37, 82-88. https://doi.org/10.1109/35.809389

[6] 3GPP (2015) Technical Specification 36.212. Tech Rep. v12.5.0.

[7] Roh W., et al. (2014) Millimeter-Wave Beamforming as an Enabling Technology for 5G Cellular Communications: Theoretical Feasibility and Prototype Results. IEEE Communications Magazine, 52, 106-113. https://doi.org/10.1109/MCOM.2014.6736750

[8] Bangerter, B., Talwar, S., Arefi, R. and Stewart, K. (2014) Networks and Devices for the 5G Era. IEEE Communications Magazine, 52, 90-96.

https://doi.org/10.1109/MCOM.2014.6736748

[9] Rappaport T.S., et al. (2013) Millimeter Wave Mobile Communications for 5G Cellular: It Will Work! IEEE Access, 1, 335-349. https://doi.org/10.1109/ACCESS.2013.2260813

[10] Chen, S. and Zhao, J. (2014) The Requirements, Challenges, and Technologies for 5G of Terrestrial Mobile Telecommunication. IEEE Communications Magazine, 52, 36-43. https://doi.org/10.1109/MCOM.2014.6815891

[11] van Nee, R. and Prasad, R. (2000) OFDM for Wireless Multimedia Communications. Artech House, Norwood.

[12] Liu, W., Lau, J. and Cheng, R.S. (1999) Considerations on Applying OFDM in a Highly Efficient Power Amplifier. IEEE Transactions on Circuits and Systems II, 46, 1329-1336.

[13] O’Neil, R. and Lopes, L.N. (1995) Envelope Variations and Spectral Splatter in Clipped Multicarrier Signals. Proceedings of 6th International Symposium on Personal, Indoor and Mobile Radio Communications, Toronto, 27-29 September 1995, 71-75. https://doi.org/10.1109/PIMRC.1995.476406

[14] Jankiraman, M. (2004) Space-Time Codes and MIMO Systems. Artech House, Norwood.

[15] Clarkson, I.V.L. and Collings, I.B. (2002) A New Joint Coding and Modulation Scheme for Channels with Clipping. Digital Signal Processing, 12, 223-241. https://doi.org/10.1006/dspr.2002.0452

[16] Bauml, R.W., Fisher, R.F. and Huber, J.B. (1996) Reducing the Peak-to-Average Power Ratio of Multicarrier Modulation by Selected Mapping. Electronics Letters, 32, 2056-2057. https://doi.org/10.1049/el:19961384

[17] Mishra, S. and Agarwal, A. (2017) Peak to Average Power Ratio Reduction in 
Sub-Carrier Index Modulated OFDM Using Selective Mapping. International Conference on Computer, Communications and Electronics, Jaipur, 168-171. https://doi.org/10.1109/COMPTELIX.2017.8003958

[18] Bae, K., Shin, C. and Powers, E.J. (2013) Performance Analysis of OFDM Systems with Selected Mapping in the Presence of Nonlinearity. IEEE Transactions on Wireless Communications, 12, 2314-2322. https://doi.org/10.1109/TWC.2013.040213.120968

[19] Muller, S.H. and Huber, J.B. (1997) OFDM with Reduced Peak-to-Average Power Ratio by Optimum Combination of Partial Transmit Sequences. Electronics Letters, 33, 368-369. https://doi.org/10.1049/el:19970266

[20] Singh, A. and Singh, H. (2016) Peak to Average Power Ratio Reduction in OFDM System Using Hybrid Technique. International Journal for Light and Electron Optics, 127, 3368-3371.

[21] Tellado, J. (2000) Peak to Average Power Reduction for Multicarrier Modulation. PhD Dissertation, Stanford University, Stanford.

[22] Andgart, N., Krongold, B.S., Ödling, P., Johansson, A. and Börjesson, P.O. (2004) PSD-Constrained PAR Reduction for DMT/OFDM. Eurasip Journal on Applied Signal Processing, No. 10, 1498-1507.

[23] Kou, Y., Lu, W.-S. and Antoniou, A. (2004) New Peak-to Average Power Ratio Reduction Algorithms for Multicarrier Communications. IEEE Transactions on Circuits and Systems I, 51, 1790-1800. https://doi.org/10.1109/TCSI.2004.834510

[24] Krongold, B.S. and Jones, D.L. (2003) PAR Reduction in OFDM via Active Constellation Extension. IEEE Transactions on Broadcasting, 49, 258-268. https://doi.org/10.1109/TBC.2003.817088

[25] Ochiai, H. and Imai, H. (1997) Block Coding Scheme Based on Complementary Sequences for Multicarrier Signals. IEICE Transactions on Fundamentals of Electronics, Communications and Computer Sciences, E80-A, 2136-2143.

[26] Davis, J.A. and Jedwab, J. (1999) Peak-to-Mean Power Control in OFDM, Golay Complementary Sequences, and Reed-Muller Codes. IEEE Transactions on Information Theory, 45, 2397-2417. https://doi.org/10.1109/18.796380

[27] Paterson, K. (2000) Generalized Reed-Muller Codes and Power Control in OFDM Modulation. IEEE Transactions on Information Theory, 46, 104-120. https://doi.org/10.1109/18.817512

[28] Li, X. and Cimini, L.J. (1997) Effects of Clipping and Filtering on the Performance of OFDM. IEEE Communications Letters, 2, 131-133.

[29] Chen, H. and Haimovich, A.M. (2003) Iterative Estimation and Cancelation of Clipping Noise Cancelation for OFDM Signals. IEEE Communications Letters, 7, 305-307. https://doi.org/10.1109/LCOMM.2003.814720

[30] Tellado, J., Hoo, L.M.C. and Ciofi, J.M. (2003) Maximum Likelihood Detection of Non-Linearly Distorted Multicarrier Symbols by Iterative Decoding. IEEE Transactions on Communications, 51, 218-228. https://doi.org/10.1109/TCOMM.2003.809289

[31] Zhu, X., Pan, W., Li, H. and Tang, Y. (2013) Simplified Approach to Optimized Iterative Clipping and Filtering for PAPR Reduction of OFDM Signals. IEEE Transactions on Communications, 61, 1891-1901. https://doi.org/10.1109/TCOMM.2013.021913.110867

[32] Tellambura, C. (2001) Computation of the Continuous-Time PAR of an OFDM Signal with BPSK Subcarriers. IEEE Communications Letters, 5, 185-187. 
https://doi.org/10.1109/4234.922754

[33] McClellan, J.H., Schafer, R.W. and Yoder, M.A. (2003) Signal Processing First. Pearson Prentice Hall, Upper Saddle River.

[34] Fraser, D. (1989) Interpolation by FFT Revisited-An Experimental Investigation. IEEE Transactions on Acoustics, Speech, and Signal Processing, 37, 665-675.

[35] Dinis, R. and Gusmao, A. (1999) On the Performance Evaluation of OFDM Transmission Using Clipping Techniques. Gateway to 21 st Century Communications Village, Amsterdam, 19-22 September 1999, 2923-2928.

[36] Jayalath, A.D.S. and Athaudage, C.R.N. (2004) On the PAR Reduction of OFDM Signals Using Multiple Signal Representation. IEEE Communications Letters, 7, 425-427. https://doi.org/10.1109/LCOMM.2004.832767

[37] Ochiai, H. and Imai, H. (2001) On the Distribution of the Peak-to-Average Power Ratio in OFDM Signals. IEEE Transactions on Communications, 49, 282-289. https://doi.org/10.1109/26.905885

[38] Saul, A. and Auer, G. (2005) Improving the Performance of Clipping Techniques for OFDM Systems by Guard Bands. 10 th OFDM International OFDM Workshop, Hamburg, 31 August-1 September 2005, 1-4.

[39] Proakis, J.G. (2004) Digital Communications. 4th Edition, McGraw Hill, New York.

[40] Baas, N.J. and Taylor, D.P. (2004) Pulse Shaping for Wireless Communication over Time or Frequency Selective Channels. IEEE Transactions on Communications, 52, 1477-1479. https://doi.org/10.1109/TCOMM.2004.833133

[41] Krongold, B.S. and Jones, D.L. (2004) An Active-Set Approach for OFDM PAR Reduction via Tone Reservation. IEEE Transactions on Signal Processing, 52, 495-509. https://doi.org/10.1109/TSP.2003.821110 